\title{
Comprehensive first-principles study of stable stacking faults in hep metals
}

\author{
Binglun Yin ${ }^{\mathrm{a}}$, Zhaoxuan Wu $\mathrm{Wu}^{\mathrm{a}, \mathrm{b}, *}, \mathrm{~W}$. A. Curtin ${ }^{\mathrm{a}}$ \\ ${ }^{a}$ Institute of Mechanical Engineering, École Polytechnique Fédérale de Lausanne, Lausanne \\ CH-1015, Switzerland \\ ${ }^{b}$ Institute of High Performance Computing, 1 Fusionopolis Way, \#16-16, Connexis, Singapore \\ 138632, Singapore
}

\begin{abstract}
The plastic deformation in hcp metals is complex, with the associated dislocation core structures and properties not well understood on many slip planes in most hcp metals. A first step in establishing the dislocation properties is to examine the stable stacking fault energy and its structure on relevant slip planes. However, this has been perplexing in the hcp structure due to additional in-plane displacements on both side of the slip plane. Here, density functional theory guided by crystal symmetry analysis is used to study all relevant stable stacking faults in 6 hcp metals ( $\mathrm{Mg}, \mathrm{Ti}, \mathrm{Zr}, \mathrm{Re}, \mathrm{Zn}, \mathrm{Cd}$ ). Specially, the stable stacking fault energy, position, and structure on the Basal, Prism I and II, Pyramidal I and II planes are determined using all-periodic supercells with full atomic relaxation. All metals show similar stacking fault position and structure as dictated by crystal symmetry, but the associated stacking fault energy, being governed by the atomic bonding, differs significantly among them. Stacking faults on all the slip planes except the Basal plane show substantial out-of-plane displacements while stacking faults on the Prism II, Pyramidal I and II planes show additional in-plane displacements, all extending to multiple atom layers. The in-plane displacements are not captured in the standard computational approach for stacking faults, and significant differences are shown in the energies of such stacking faults between the standard approach and fully-relaxed case. The existence of well-defined stable stacking fault on the Pyramidal planes suggests zonal dislocations are unlikely. Calculations on the equilibrium partial separation further suggests $\langle\mathbf{c}+\mathbf{a}\rangle$ dissociation into three
\end{abstract}

\footnotetext{
${ }^{*}$ Corresponding author

Email address: zhaoxuan.wu@epfl.ch (Zhaoxuan Wu)
} 
partials on the Pyramidal I plane is unlikely and $\langle\mathbf{c}\rangle$ dissociation on Prism planes is unlikely to be stable against climb-dissociation onto the Basal planes in these metals.

Keywords: HCP, Stacking Fault, ab initio Calculations, Dislocation Dissociation

\section{Introduction}

The generalized stacking fault energy (GSFE) $\gamma$-surface is one of the most fundamental properties in crystalline materials. Since first introduced by Vitek [1], it has achieved impressive success in both explaining and predicting dislocation core structures and associated behavior on close-packed planes of face-centered cubic (fcc), body-centered cubic (bcc) and hexagonal close-packed (hcp) metals [2]. The standard $\gamma$-surface, $\gamma(\mathbf{t})$, is defined as the excess energy of the crystal when atoms on one side of a crystallographic plane are displaced by a rigid-inplane shift $\mathbf{t}$ relative to the atoms on the other side, followed by relaxation of displacements in the out-of-plane direction. On the $\gamma$-surface, the absolute energy minima correspond to shifts by the lattice translation vectors $\mathbf{a}_{1}$ or $\mathbf{a}_{2}$ of the crystallographic plane, which re-establish the perfect lattice structure. The $\gamma$-surface is thus periodic with periodicity defined by $\mathbf{a}_{1}$ and $\mathbf{a}_{2}$. Plastic deformation generally occurs by relative slip over one of the lattice translation vectors, which are equal to the Burgers vector $\mathbf{b}$ of the associated dislocations. The microscopic slip process occurs along a minimum energy path (MEP) between the absolute minima. The energy profile along the MEP, the $\gamma$-line, thus measures the relative ease of the slip, and determines key parameters dictating fundamental plastic properties of crystalline materials. The maximum slope along the $\gamma$-line gives the ideal shear strength, the maximum energy $\gamma_{\text {usf }}$ governs dislocation nucleation at stress concentrations such as crack tips [3], and metastable points (local minima) correspond to stable stacking faults (SFs) with energies $\gamma_{\text {sf }}$ that control dislocation core dissociation, Peierls stress, dislocation energy, and primary slip planes $[4,5]$. The general shape of the $\gamma$-surface is strongly dictated by crystal symmetry, and thus should be similar in materials of the same crystal structure. Given its importance and general applicability, the $\gamma$-surface has been calculated in many metals and alloys using a wide range of computational models ranging from pair, many-body, bond-order, and tight-binding [6] potentials to ab initio density functional theory (DFT) calculations.

The standard approach for computing $\gamma(\mathbf{t})$, involving deviations from the perfect crystalline structure only in the out-of-plane direction, is less accurate when 
applied to corrugated faults, such as occurs for SFs on Pyramidal planes of the hcp structure. Previous studies reported inconsistencies between the stable SF predicted by the standard $\gamma$-lines and the SF and dislocation dissociations found by direct atomistic calculations [7, 8]. A recent DFT study on $\langle\mathbf{c}+\mathbf{a}\rangle$ dislocation core structures in $\mathrm{Mg}$ also shows a significant difference in partial core separation between the direct simulations and the predictions of elasticity theory using the stable SF energy computed by the standard approach [9]. Morris et al. [10] proposed a new procedure, allowing in-plane relaxation of atoms, to calculate the $\gamma$ line of the hcp Pyramidal planes. Applying the new method to systems described by empirical potentials, the new $\gamma$-lines showed both qualitative and quantitative differences with those of the standard approach. The necessity of in-plane relaxation was also recognized in a few recent DFT calculations of the SF energy on some slip planes in $\mathrm{Mg}$ [11], Ti [12, 13] and $\mathrm{Zr}$ [14, 15, 16, 17], again showing strong effects of full relaxation on the predicted SF. While SF energy calculations with full relaxations are emerging, there has been no complete examination of the true stable SF energies, positions, and structures on all relevant slip planes across the family of hcp metals. Accurate SF energies and structures on Prism and Pyramidal planes are particularly important for understanding/prediction of the core structure, dissociation, and stability of $\langle\mathbf{c}\rangle$ and $\langle\mathbf{c}+\mathbf{a}\rangle$ dislocations, for which direct DFT simulations remain challenging although not impossible [16, 9, 18, 19]. In this work, we perform DFT calculations with full atomic (ionic) relaxation to find all the stable SFs on all the slip planes relevant to plastic deformation of hcp metals. In particular, we provide a systematic examination of Basal, Prism I and II, and Pyr. I and II planes (shown in Fig. 1) in 6 hcp metals (Mg, Ti, Zr, $\mathrm{Re}, \mathrm{Zn}, \mathrm{Cd})$. Our results show that all 6 metals show similar stable SF positions and structures, despite the differences in their electronic structures. At the stable SFs, out-of-plane displacements are substantial in all cases except on the Basal plane, while in-plane displacements are substantial on the Prism II and Pyr. I and II planes. The atomic displacements decay quickly within a few layers of atoms on either side of the slip plane and the displacements in the slip direction are localized to the slip plane. The existence of clear stable SF and well-defined SF structures suggest that zonal dislocations $[20,21]$ are unlikely on any of these slip planes. Furthermore, atomic displacements on the Prism and Pyramidal planes correspond to some form of atomic shuffling, making slip on these planes relatively difficult as compared to slip on the Basal plane; i.e. the critical resolved shear stresses for dislocation glide on these Prism and Pyramidal planes is expected to be rather larger than that for Basal slip, in materials where Basal screw dislocations are stable. Using the obtained SF information combined with elastic- 
ity theory, we further determine the equilibrium partial separations of all relevant dislocations. Our results show that dissociation of $\langle\mathbf{c}+\mathbf{a}\rangle$ dislocations into three partials on the Pyr. I plane and glide dissociations of $\langle\mathbf{c}\rangle$ dislocations on the Prism planes are highly unlikely in these metals.

The remainder of this paper is organized as follows. We first establish the possible stable SF vectors on all the relevant hcp slip planes based on symmetry and a hard sphere model. The DFT methods are then described in detail in Section 3. The results for SF energies, actual SF fault vectors, and SF structures are presented in Section 4. Section 5 discusses implications of our results, including dislocation dissociations.

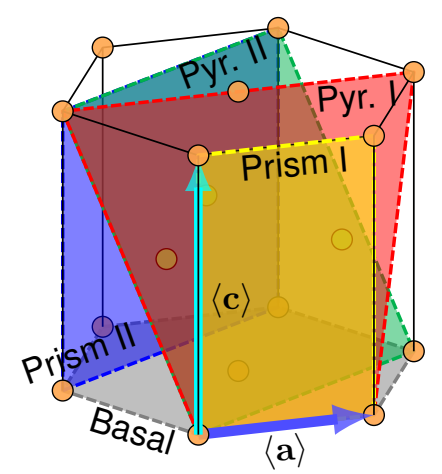

Figure 1: Schematic showing the Basal, Prism I and II, and Pyramidal I and II planes in the hcp unit cell.

\section{Stacking faults based on symmetry in hcp crystals}

For computations, the initial choice of the in-plane shift $\mathbf{t}^{\prime}$ is important. If the choice is not close to the true metastable fault vector, the initial structure can relax back to a uniformly-strained perfect crystal. Since DFT computations are very costly, a good initial choice for $\mathbf{t}^{\prime}$ is essential. We therefore first determine possible $\mathbf{t}^{\prime}$ based on energy extrema on the $\gamma$-surface using crystal symmetry and a hard sphere model(see Ref. $[8,2]$ on similar analysis on some planes in hcp structure). Figure 2 and Fig. 3 show the atomic structure and the corresponding slip vectors for the 5 slip planes considered in the current analysis.

On the Basal plane (Fig. 2a), an energy minimum must exist at the intersection of the three mirror planes (indicated by the $m_{i}$ lines), i.e. at $\mathbf{t}=1 / 3[\overline{1} 100]$ or $\left(\mathbf{a}_{1} / 2,\left(\mathbf{a}_{2} \cdot \mathbf{e}_{2}\right) \mathbf{e}_{2} / 3\right)$, where the definitions of $\mathbf{a}_{i}$ and $\mathbf{e}_{i}$ are shown in Fig. 2. This 

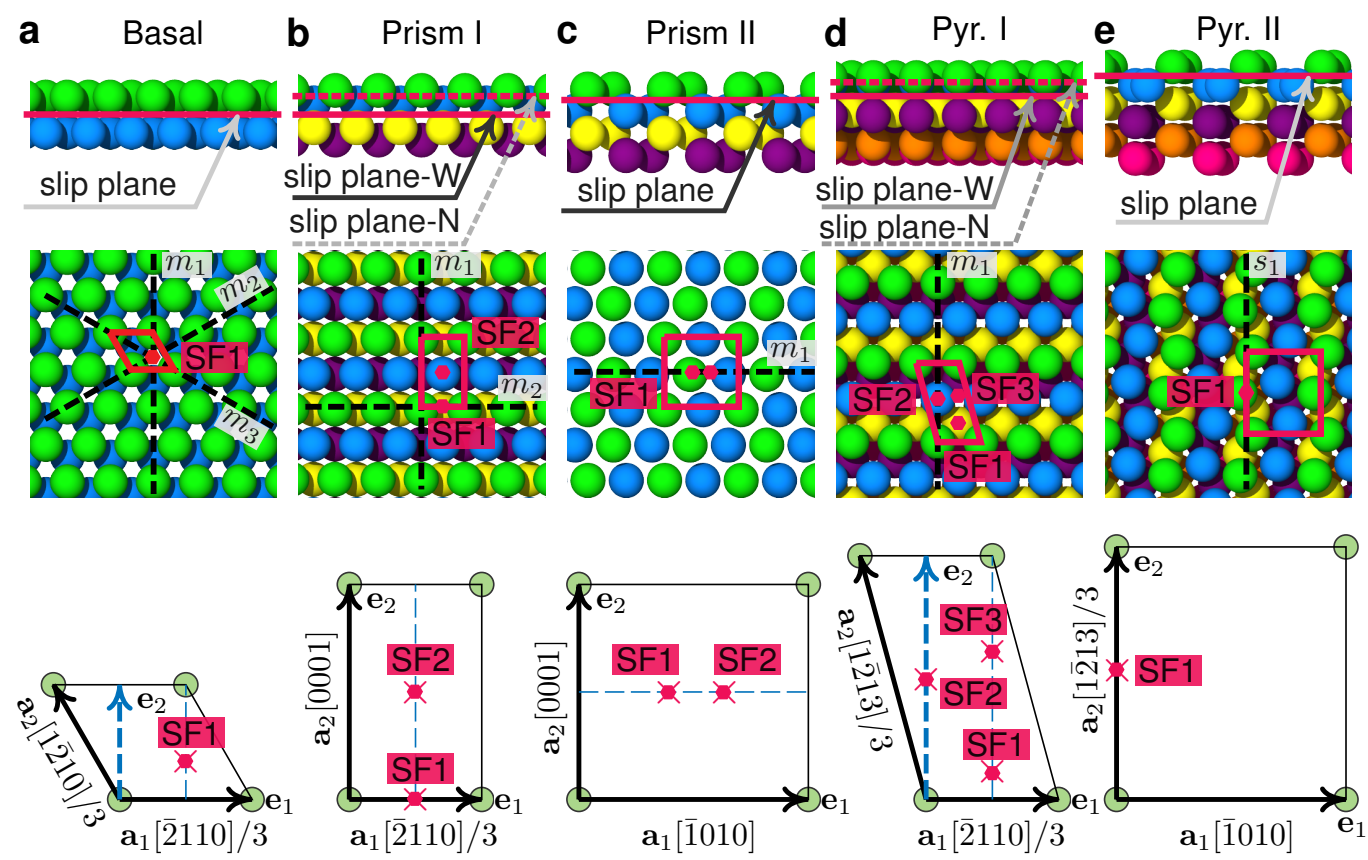

Figure 2: Atomic structure and stacking fault energy extrema on (a) Basal, (b) Prism I, (c) Prism II, (d) Pyramidal I and (e) Pyramidal II planes. In (b) and (d), the wide and narrow planes are marked by the solid and dashed lines. The white dotted lines denoted by $m_{i}$ and $s_{i}$ are the mirror symmetry plane and glide plane, respectively. In the third row, $\mathbf{a}_{1}$ and $\mathbf{a}_{2}$ are the primitive vectors in the respective crystallographic plane, $\mathbf{e}_{1}$ and $\mathbf{e}_{2}$ are the unit vectors of the Cartesian coordinate system. 
minimum is the so-called $I_{2} \mathrm{SF}$ relevant to $\langle\mathbf{a}\rangle$ dislocation dissociations on the Basal plane. Slip to the $I_{2}$ SF should be accompanied by out-of-plane atomic movement only, as shown in Fig. 3a.

The Prism I plane has two layers of atoms and thus two slip planes of different structure and interplanar spacing [22, 17], as shown in Fig. 2b. The narrowly and widely spaced slip planes are referred to as the "narrow" and "wide" planes and are denoted by "-N" and "-W", respectively. On both the narrow and wide planes, at least three energy extrema must exist at the intersection of the mirror planes, i.e. at SF1 ( $\left.\mathbf{a}_{1} / 2,0\right), \operatorname{SF} 2\left(\mathbf{a}_{1} / 2, \mathbf{a}_{2} / 2\right)$ and SF3 $\left(0, \mathbf{a}_{2} / 2\right)$, respectively. SF1 and SF2 are relevant to $\langle\mathbf{a}\rangle$ and $\langle\mathbf{c}\rangle$ dislocation dissociations. SF3 should be a maximum and thus not relevant to dislocation dissociations, and so is not considered in the current study [2,23]. Slip on the narrow plane to SF1 or SF2 should not be energetically favorable, as both cases bring near-neighbor atoms (green and blue atoms) into close proximity. In contrast, SF1 along the wide plane, as shown in Fig. 3b, can be stable if multibody (angular, second-neighbor, etc.) forces exist (the blue/green atoms could be stabilized by the yellow/purple atoms below). Slip to SF1 should also be accompanied by out-of-plane atomic movement only. SF2 on the wide plane should also be stable, but both in-plane and out-of-plane atomic movements are expected along the slip path (see Fig. 3c). On the Prism II plane (Fig. 2c), energy extrema must exist along ( $\left.m \mathbf{a}_{1}, \mathbf{a}_{2} / 2\right)$, which is relevant to the $\langle\mathbf{c}\rangle$ dissociation on that plane. Slips on the Prism II plane to SF1 or SF2 are equivalent, as shown in Fig. 3d and e, and should involve both in-plane and out-of-plane atomic movements.

The Pyr. I plane also has two layers of atoms and slip can thus take place on either the "wide" or "narrow" planes (see Fig. 2d). On both slip planes, there are at least three possible energy extrema. SF1 at $\left(\mathbf{a}_{1} / 2, m\left(\mathbf{a}_{2} \cdot \mathbf{e}_{2}\right) \mathbf{e}_{2}\right)$ is relevant to $\langle\mathbf{a}\rangle$ dislocation dissociation. SF2 and SF3 at $\left(0, n\left(\mathbf{a}_{2} \cdot \mathbf{e}_{2}\right) \mathbf{e}_{2}\right)$ and $\left(\mathbf{a}_{1} / 2, m\left(\mathbf{a}_{2} \cdot \mathbf{e}_{2}\right) \mathbf{e}_{2}\right)$ are relevant to $\langle\mathbf{c}+\mathbf{a}\rangle$ dissociation. SF1 on the narrow plane should be stable and energetically more favorable than on the wide plane (see Fig. 3f). Slip to SF1 should be accompanied by both in-plane and out-of-plane atomic movements. Slip to SF2 or SF3 is associated to $\langle\mathbf{c}+\mathbf{a}\rangle$ dislocations and should be accompanied by out-of-plane and in-plane atomic movement in the slip direction only (Fig. $3 \mathrm{~g}$ and h). The slip process is energetically more favorable on the wide plane and is highly unfavorable on the narrow plane due to the close proximity of near-neighbor atoms (green and blue atoms). On the Pyr. II plane, at least one energy extremum must exist at $\operatorname{SF} 1\left(0, m \mathbf{a}_{2}\right)$. This fault is relevant to $\langle\mathbf{c}+\mathbf{a}\rangle$ dissociations. Slip to SF1 should be accompanied by both in-plane and out-of-plane atomic movements and involves close interaction between the nearest-neighbor atoms (Fig. 3i and 

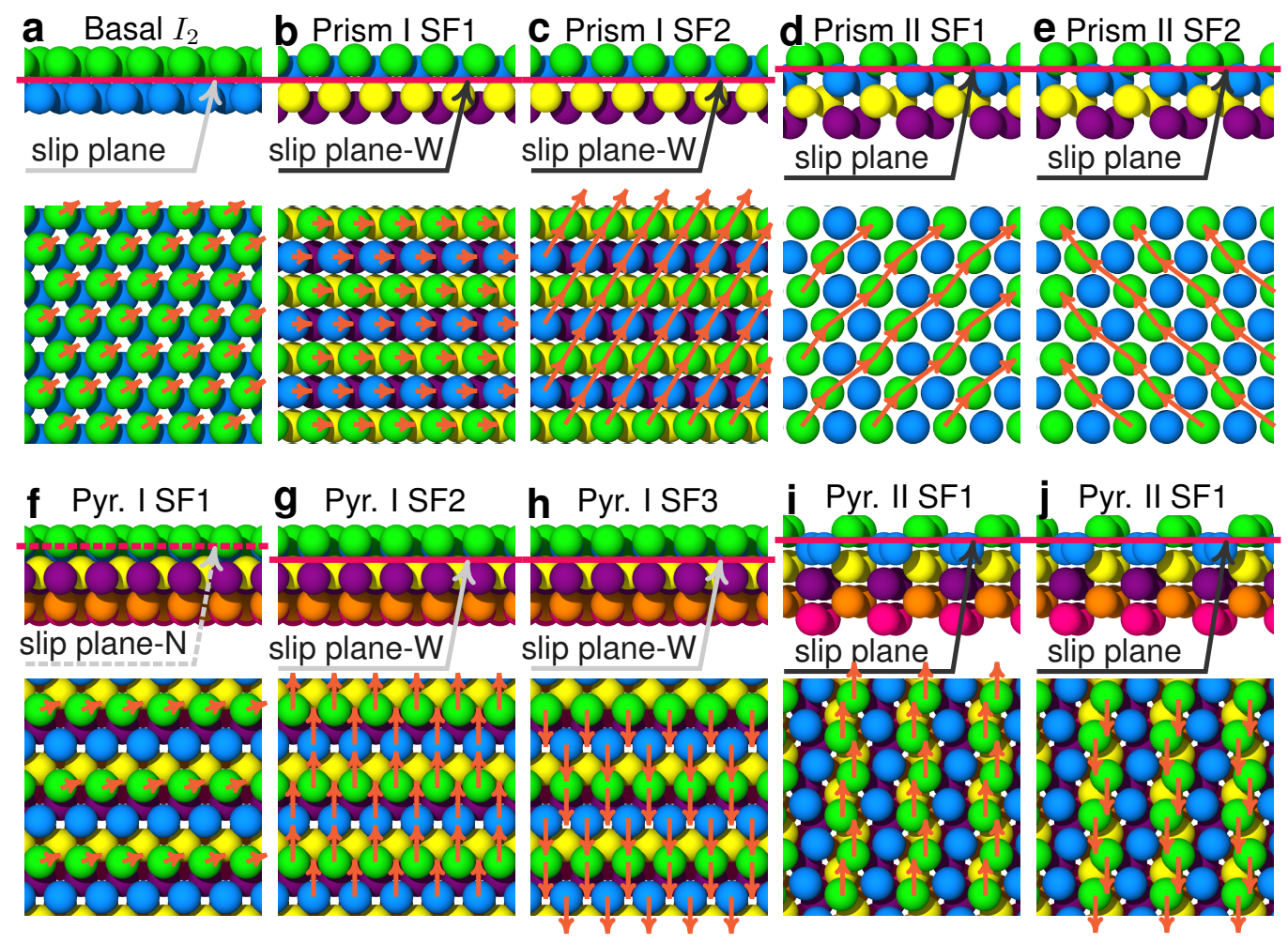

Figure 3: Slip vector for (a) Basal $I_{2}$, (b) Prism I SF1, (c) Prism I SF2, (d) Prism II SF1, (e) Prism II SF2, (f) Pyr. I SF1, (g) Pyr. I SF2, (h) Pyr. I SF3, (i) Pyr. II SF1 and (j) Pyr. II SF1 in opposite direction. 
j). Furthermore, on both Pyr. I and II planes, slip associated with $\langle\mathbf{c}+\mathbf{a}\rangle$ is direction-dependent (see Fig. 3g-j) and the associated $\gamma$-line is thus unsymmetric in general.

We note that the above analysis only uses crystal symmetry and can only provide qualitative assessment on stacking fault position and atomic movement along the slip path. The details of the electronic structure can strongly influence the energetics and kinetics along the slip path, and have to be taken into account for quantitative analysis. This is pursued in the DFT calculations presented below.

\section{DFT methodology and simulation geometries}

The SF positions determined above are used as the initial in-plane shifts $\mathbf{t}^{\prime}$ for DFT calculations. DFT calculations are performed using the Vienna $A b$ initio Simulation Package (VASP) [24, 25] with a plane-wave spin-free basis set. The generalized gradient approximation (GGA) with the Perdew-Burke-Ernzerhof (PBE) parametrization [26] is used for the exchange and correlation functionals. The core electrons are replaced by the projector augmented wave (PAW) pseudopotentials [27] with the valence states shown in Table 1. A second-order MethfesselPaxton method [28] with smearing parameter $0.2 \mathrm{eV}$ is used to smoothen the partial electron occupancies. Ionic positions are relaxed toward local equilibrium states, with convergence deemed achieved when the maximum ionic force is less than $1 \mathrm{meV} / \AA$. For each element, the appropriate plane-wave cutoff energy and $\Gamma$-centered Monkhorst-Pack [29] $k$-point sampling mesh are carefully established through convergence tests on the basal $I_{2}$ SF energy and bulk stress state of the 2 -atoms hcp primitive cell; the values used here are shown in Table. 1 . The $k$ point mesh for larger supercells is adjusted to give a very close $k$-point density. Using the above parameters, the predicted lattice constants and elastic constants (calculated by fitting computed total energies versus strain) are in good agreement with experiments, as shown in Table 2.

Table 1: Valence states, plane wave energy cutoffs, and k-mesh densities used in the DFT calculations.

\begin{tabular}{lllllll}
\hline & $\mathbf{M g}$ & $\mathbf{T i}$ & $\mathbf{Z r}$ & $\mathbf{R e}$ & $\mathbf{Z n}$ & $\mathbf{C d}$ \\
\hline valence states & $3 s$ & $4 s, 3 d$ & $5 s, 4 d$ & $6 s, 5 d$ & $4 s, 3 d$ & $5 s, 4 d$ \\
$E_{\text {cutoff }}(\mathrm{eV})$ & 350 & 350 & 350 & 650 & 500 & 500 \\
$k$-mesh (primitive cell) & $36 \times 36 \times 19$ & $39 \times 39 \times 22$ & $36 \times 36 \times 19$ & $37 \times 37 \times 20$ & $39 \times 39 \times 18$ & $38 \times 38 \times 17$ \\
\hline
\end{tabular}

The simulation supercells for SF energy calculations are constructed using the above determined lattice constants. To avoid spurious effects due to free surfaces 
Table 2: Lattice parameter, $c / a$ ratio and elastic constants calculated by DFT and comparison with experimental values.

\begin{tabular}{|c|c|c|c|c|c|c|c|c|c|c|c|c|}
\hline & \multicolumn{2}{|c|}{ Mg } & \multicolumn{2}{|c|}{$\mathbf{T i}$} & \multicolumn{2}{|c|}{$\mathbf{Z r}$} & \multicolumn{2}{|c|}{$\mathbf{R e}$} & \multicolumn{2}{|c|}{$\mathbf{Z n}$} & \multicolumn{2}{|c|}{ Cd } \\
\hline & DFT & Exp & DFT & Exp & DFT & Exp & DFT & Exp & DFT & Exp & DFT & Exp \\
\hline$a(\AA)$ & 3.189 & $3.186^{\mathrm{a}}$ & 2.924 & $2.947^{\mathrm{a}}$ & 3.235 & $3.228^{\mathrm{a}}$ & 2.774 & $2.760^{\mathrm{a}}$ & 2.655 & $2.647^{\mathrm{a}}$ & 3.031 & $2.957^{\mathrm{a}}$ \\
\hline$c / a$ & 1.627 & $1.624^{\mathrm{a}}$ & 1.582 & $1.586^{\mathrm{a}}$ & 1.598 & $1.593^{\mathrm{a}}$ & 1.616 & $1.615^{\mathrm{a}}$ & 1.891 & $1.856^{\mathrm{a}}$ & 1.911 & $1.886^{\mathrm{a}}$ \\
\hline$C_{11}(\mathrm{GPa})$ & 61 & $63^{\mathrm{b}}$ & 177 & $176^{\mathrm{b}}$ & 147 & $155^{\mathrm{b}}$ & 616 & $634^{\mathrm{b}}$ & 190 & $179^{\mathrm{b}}$ & 83 & $129^{\mathrm{b}}$ \\
\hline$C_{12}(\mathrm{GPa})$ & 26 & $26^{\mathrm{b}}$ & 90 & $87^{b}$ & 70 & $67^{\mathrm{b}}$ & 278 & $266^{\mathrm{b}}$ & 49 & $38^{\mathrm{b}}$ & 43 & $40^{\mathrm{b}}$ \\
\hline$C_{13}(\mathrm{GPa})$ & 20 & $22^{\mathrm{b}}$ & 84 & $68^{\mathrm{b}}$ & 71 & $65^{\mathrm{b}}$ & 226 & $202^{\mathrm{b}}$ & 52 & $55^{\mathrm{b}}$ & 30 & $41^{\mathrm{b}}$ \\
\hline$C_{33}(\mathrm{GPa})$ & 63 & $66^{\mathrm{b}}$ & 189 & $191^{\mathrm{b}}$ & 164 & $173^{\mathrm{b}}$ & 671 & $702^{\mathrm{b}}$ & 70 & $69^{\mathrm{b}}$ & 41 & $57^{\mathrm{b}}$ \\
\hline$C_{44}(\mathrm{GPa})$ & 18 & $18^{\mathrm{b}}$ & 40 & $51^{\mathrm{b}}$ & 25 & $36^{b}$ & 161 & $169^{\mathrm{b}}$ & 47 & $46^{\mathrm{b}}$ & 13 & $24^{\mathrm{b}}$ \\
\hline
\end{tabular}

${ }^{\mathrm{a}}$ Lattice constants calculated based on material density at $4 \mathrm{~K}$ and room temperature $c / a$ ratio [30, 31].

${ }^{\mathrm{b}}$ Elastic constants measured at $4 \mathrm{~K}$ in experiments [31].

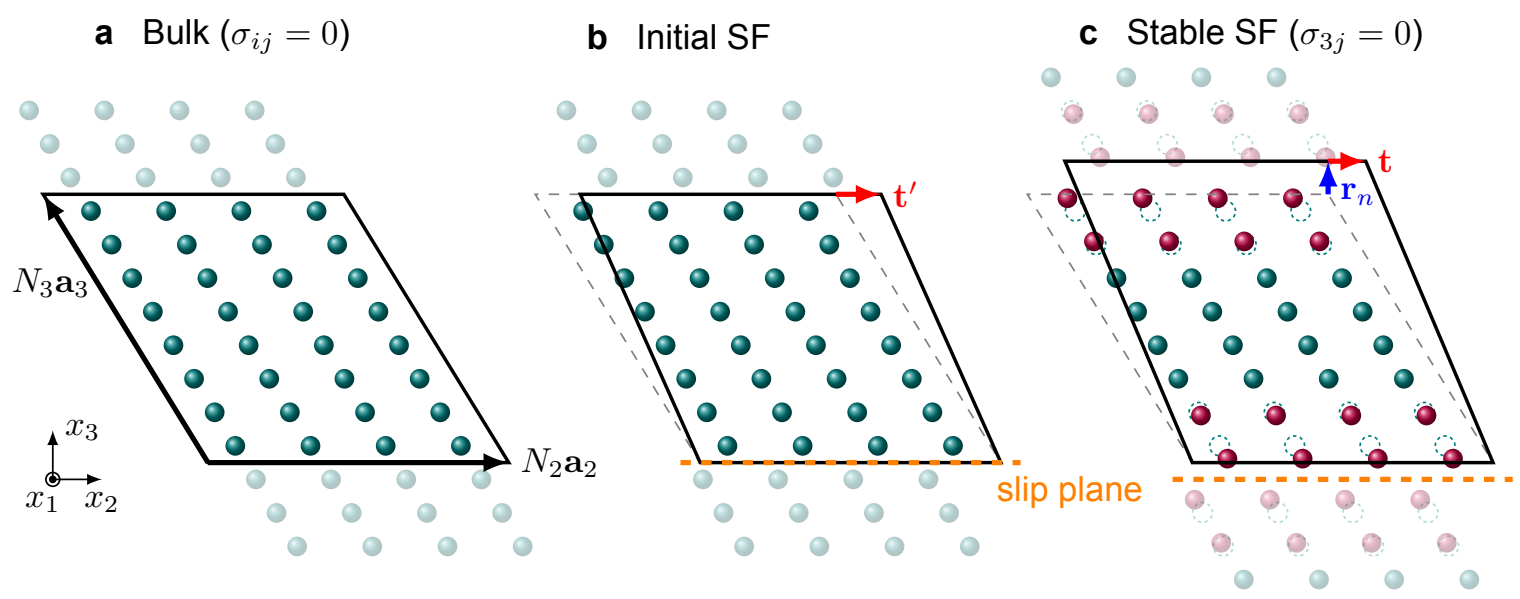

Figure 4: (a) Periodic supercell of a perfect lattice with the lattice translation vectors $\mathbf{a}_{1}$ and $\mathbf{a}_{2}$ of the crystallographic slip plane in the $x_{1}-x_{2}$ plane. (b) The initial (ideal) stacking fault created by tilting the supercell repeat vector $N_{3} \mathbf{a}_{3}$ to $N_{3} \mathbf{a}_{3}+\mathbf{t}^{\prime}$ while keeping $N_{1} \mathbf{a}_{1}, N_{2} \mathbf{a}_{2}$ and all the atoms fixed. (c) Fully relaxed stacking fault in the periodic supercell. The dotted and solid circles represent atom positions before and after relaxation. 
(e.g., Friedel oscillations [32]) and to reduce computational cost, supercells periodic in all directions are used. Two types of supercells are necessary: one for the intrinsic $I_{1}$ and extrinsic $E$ faults on the Basal plane, which can not be formed through a single shear operation; the other for the basal $I_{2}$ and SFs on the Prism and Pyramidal planes, which can be formed through a single shear operation. The supercells used for the $I_{1}$ and $E$ faults have 40 atom layers in the $x_{3}$ (out-of-plane) direction arranged as $(A B)_{10}-(A C)_{10}$ and $(A B)_{10}-C-(A B)_{9}-C$, respectively, where $\mathrm{A}, \mathrm{B}$, and $\mathrm{C}$ denote the relative stacking sequence of (111) planes in fcc crystals (ABCABC...) and basal planes in hcp crystals (ABAB...) [5]. Because periodic boundary conditions are used, two identical stacking faults are formed within each supercell. For all other SFs, the tilted-cell method [33, 34, 35] is used. We start with a primitive cell of the perfect lattice with slip plane lattice vectors $\mathbf{a}_{1}$ and $\mathbf{a}_{2}$ in the $\left(x_{1}-x_{2}\right)$ plane and $\mathbf{a}_{3}$ out of the SF plane, as shown in Fig. $4 \mathbf{a}\left(\mathbf{a}_{1}, \mathbf{a}_{2}\right.$ and $\mathbf{a}_{3}$ are not orthogonal in general). The primitive cell is repeated in $\mathbf{a}_{1}, \mathbf{a}_{2}$ and $\mathbf{a}_{3}$ directions to obtain a parallelepiped supercell with periodic repeat vectors $\left(N_{1} \mathbf{a}_{1}\right.$, $\left.N_{2} \mathbf{a}_{2}, N_{3} \mathbf{a}_{3}\right)$. To create a SF, the supercell repeat vector $N_{3} \mathbf{a}_{3}$ is tilted to $N_{3} \mathbf{a}_{3}+\mathbf{t}^{\prime}$ while keeping $N_{1} \mathbf{a}_{1}, N_{2} \mathbf{a}_{2}$ and all the atoms fixed. This tilt procedure does not move any atoms, but effectively shifts the periodic image in the $N_{3} \mathbf{a}_{3}$ direction by $\pm \mathbf{t}^{\prime}$, thus creating a preliminary (ideal) single-layer SF at the top and bottom boundary of the supercell (see Fig. 4b). The entire system is then relaxed to a local equilibrium configuration through a combination of ionic displacements and changes in the third supercell repeat vector $N_{3} \mathbf{a}_{3}+\mathbf{t}^{\prime}$ so that all stress components $\sigma_{3 j}$ are zero (see Fig. 4c). During the relaxation, the in-plane lattices $\left(N_{1} \mathbf{a}_{1}, N_{2} \mathbf{a}_{2}\right)$ are fixed to the bulk values, in order to achieve results that are independent of $N_{3}$ (otherwise, the intrinsic stresses of the SF would be balanced by opposite stresses distributed through the remainder of the cell, and the associated elastic energy would depend on the cell size). After full relaxation, the relaxed supercell repeat vector in the third direction is $N_{3} \mathbf{a}_{3}+\mathbf{t}+\mathbf{r}_{n}$ where $\mathbf{t}$ is the in-plane stable SF vector and $\mathbf{r}_{n}$ is the net relaxation perpendicular to the SF plane. This procedure results in the true stable SF structure, vector, and energy relevant to dislocation behavior. Since the SF can possibly have substantial relaxations away from the slip plane, it is important to use large $N_{3}$ to avoid spurious interactions between the periodic images of the SF. In the current study and unless otherwise stated, $N_{1}=N_{2}=1$ and $N_{3}=10$ is used for each case.

The atomic displacement $\mathbf{u}$ is calculated as

$$
\mathbf{u}=\mathbf{X}-\mathbf{x}
$$

where $\mathrm{X}$ is atom position after full relaxation (Fig. 4c) and $\mathrm{x}$ is atom position at 
the ideal stacking fault configuration before relaxation (Fig. 4b), i.e. the perfect lattice position in the tilted-cell method. It is the displacement in addition to $t$ required to form the ideal stacking fault in the standard method. $\mathbf{u}$ thus measures atomic displacement from the ideal stacking fault. If the ideal stacking fault configuration is stable, $\mathbf{u}=0$. In the standard stacking fault calculation method, the in-plane displacement is constrained, i.e. $u_{1}=u_{2}=0$ and only $u_{3}$ is allowed to vary.

\section{DFT-calculated stacking fault vectors, energies, and structures}

Table 3 shows the results for SF energy and fault vector as calculated here by DFT, along with the SF energies reported in previous DFT studies. On the Basal plane, all these hcp metals have an $I_{2} \mathrm{SF}$ at $\left(\mathbf{a}_{1} / 2,\left(\mathbf{a}_{2} \cdot \mathbf{e}_{2}\right) \mathbf{e}_{2} / 3\right)$, as expected from crystal symmetry. The ratio of the $I_{1}, I_{2}$ and Extrinsic SF energies is approximately equal to the ratio of atoms with second nearest-neighbor symmetry broken in the respective faults, i.e., $1: 2: 3$. The results here using full atomic relaxation are also in good agreement with available DFT data calculated using the standard procedure. This indicates that in-plane relaxation has small effects on SF on the Basal plane, i.e. negligible in-plane relaxations are expected, consistent with the crystal symmetry analysis.

On the Prism I plane, only Ti, $\mathrm{Zr}$ and Re show a stable SF at $\left(\mathbf{a}_{1} / 2,0\right)$. For $\mathrm{Mg}, \mathrm{Zn}$, and $\mathrm{Cd}$, we therefore compute the unstable SF energy at $\left(\mathbf{a}_{1} / 2,0\right)$ by symmetry-constrained relaxation, which prevents the system from moving away from the extremal symmetry point. The unstable SF in these metals can serve as a proxy for possible stable SFs that may exist but that are not resolved in the current DFT calculations [38]. The Basal $I_{2}$ energy is substantially lower than the SF1 energy on the Prism plane in $\mathrm{Mg}, \mathrm{Re}, \mathrm{Zn}$ and $\mathrm{Cd}$. In contrast, the SF1 energy on the Prism plane is lower than the energy of the $I_{2}$ fault on the Basal plane in Ti and $\mathrm{Zr}$. This suggests that $\langle\mathbf{a}\rangle$ slip is dominant on the Basal plane in $\mathrm{Mg}, \mathrm{Re}, \mathrm{Zn}$ and $\mathrm{Cd}$ and on the Prism I plane in $\mathrm{Ti}$ and $\mathrm{Zr}$, consistent with broad experimental observations. In addition, all metals except $\mathrm{Cd}$ have a stable SF (with a relatively high energy) at or close to $\left(\mathbf{a}_{1} / 2, \mathbf{a}_{2} / 2\right)$, suggesting a possible $\langle\mathbf{c}\rangle$ dissociation on this plane. On the Prism II plane, all metals show a stable SF with relatively low energy, indicating favorable $\langle\mathbf{c}\rangle$ dissociation on this plane. In addition, the SF1 energy on the Prism I plane calculated using full relaxation is again in good agreement with available DFT data computed using the standard method, suggesting small in-plane relaxation at this SF. In contrast, large differences are seen for the SF1 energy on the Prism II plane, indicating substantial in-plane relaxation. 
Table 3: Stacking fault energies and positions as calculated by DFT. $\gamma_{\mathrm{sf}}$ and $\mathbf{t}$ are in the units of $\mathrm{mJ} / \mathrm{m}^{2}$ and $\left(\mathbf{a}_{1} \cdot \mathbf{e}_{1}\right.$, $\mathbf{a}_{2} \cdot \mathbf{e}_{2}$, see Fig. 2), respectively. Results from related literature calculations are shown below the present results in each case, with results in plain font calculated using full relaxation and results in italic font calculated using the standard method. Number followed by $[\mathrm{u}]$ indicates unstable stacking fault in DFT calculations and the quoted energy is obtained using symmetry-constrained relaxation. In Cd, the structure of SF2 on Prism I-W (denoted by $[\mathrm{x}]$ ) is not stable with $N_{3}=10$ in the current DFT calculations.

\begin{tabular}{|c|c|c|c|c|c|c|c|c|}
\hline & & & Mg & $\mathbf{T i}$ & $\mathbf{Z r}$ & $\operatorname{Re}$ & $\mathbf{Z n}$ & Cd \\
\hline \multirow{8}{*}{ Basal } & \multirow[b]{2}{*}{$I_{1}$} & & 18 & 172 & 149 & 54 & 48 & 17 \\
\hline & & $\gamma_{\mathrm{sf}}$ & & $149^{\mathrm{a}}, 180^{\mathrm{b}}$ & $168^{\mathrm{b}}$ & & & \\
\hline & \multirow{2}{*}{$E$} & $\gamma$ & 54 & 424 & 284 & 351 & 150 & 41 \\
\hline & & $\gamma_{\mathrm{sf}}$ & & $353^{\mathrm{a}}, 433^{\mathrm{b}}$ & $300^{\mathrm{b}}$ & & & \\
\hline & \multirow{4}{*}{$I_{2}$} & $\mathrm{t}$ & $(-0.501,0.334)$ & $(-0.500,0.333)$ & $(-0.499,0.333)$ & $(-0.500,0.334)$ & $(-0.493,0.329)$ & $(-0.481,0.321)$ \\
\hline & & & 34 & 306 & 224 & 206 & 109 & 29 \\
\hline & & & $34^{\mathrm{c}}, 21^{\mathrm{e}}$ & $259^{\mathrm{a}}, 336^{\mathrm{b}}, 287^{\mathrm{e}}$ & $213^{\mathrm{b}}, 223^{\mathrm{e}}, 227^{\mathrm{k}}$ & & $102^{\mathrm{e}}$ & \\
\hline & & & & $\begin{array}{l}307^{\mathrm{f}}, 320^{\mathrm{g}}, 292^{\mathrm{h}} \\
306^{\mathrm{i}}, 291^{\mathrm{j}}\end{array}$ & $213^{1}, 200^{\mathrm{j}}$ & & & \\
\hline \multirow{5}{*}{ Prism I-Y } & \multirow{3}{*}{ SF1 } & $\mathbf{t}$ & $(0.500,0.000)$ & $(0.500,0.000)$ & $(0.499,0.000)$ & $(0.500,0.000)$ & $(0.500,0.000)$ & $(0.500,0.000)$ \\
\hline & & & $212[u]$ & 214 & 183 & 933 & $502[\mathrm{u}]$ & $238[u]$ \\
\hline & & $\gamma_{\mathrm{sf}}$ & $218,218^{\mathrm{d}}$ & $\begin{array}{l}206^{\mathrm{b}}, 220^{\mathrm{h}}, 203^{\mathrm{i}} \\
256^{\mathrm{m}}, 174^{\mathrm{j}}\end{array}$ & $\begin{array}{l}166^{\mathrm{b}}, 197^{\mathrm{k}}, 211^{\mathrm{l}}, \\
145^{\mathrm{j}}\end{array}$ & & & \\
\hline & $\mathrm{SF} 2$ & $\mathbf{t}$ & $(0.492,0.498)$ & $(0.500,0.500)$ & $(0.500,0.500)$ & $(0.500,0.500)$ & $(0.507,0.519)$ & {$[\mathrm{x}]$} \\
\hline & $\mathrm{SF} 2$ & $\gamma_{\mathrm{sf}}$ & 383 & 1034 & 719 & 2111 & 305 & {$[\mathrm{x}]$} \\
\hline \multirow{3}{*}{ Prism II } & \multirow{3}{*}{ SF1 } & & $(0.501,0.502)$ & $(0.451,0.500)$ & $(0.446,0.500)$ & $(0.500,0.500)$ & $(0.498,0.497)$ & $(0.499,0.497)$ \\
\hline & & & 183 & 395 & 331 & 1361 & 120 & 71 \\
\hline & & & & $606^{\mathrm{b}}$ & $534^{\mathrm{b}}$ & & & \\
\hline \multirow{3}{*}{ Pyr. I-N } & \multirow{3}{*}{$\mathrm{SF} 1$} & $\mathrm{t}$ & $(0.503,-0.116)$ & $(0.500,-0.089)$ & $(0.500,-0.106)$ & $(0.500,-0.112)$ & $(0.506,0.025)$ & $(0.489,0.034)$ \\
\hline & & & 165 & 200 & 161 & 653 & 328 & 171 \\
\hline & & & & $205^{\mathrm{i}}, 227^{\mathrm{m}}$ & $163^{\mathrm{n}}$ & & & \\
\hline \multirow{5}{*}{ Pyr. I-W } & \multirow{3}{*}{$\mathrm{SF} 2$} & $\mathrm{t}$ & $(0.000,0.403)$ & $(0.000,0.435)$ & $(0.000,0.430)$ & $(0.000,0.421)$ & $(0.005,0.487)$ & $(0.000,0.572)$ \\
\hline & & & 161 & 134 & 103 & 721 & 341 & $181[\mathrm{u}]$ \\
\hline & & $\gamma_{\mathrm{sf}}$ & & $148^{\mathrm{i}}$ & & & & \\
\hline & $\mathrm{SF}_{3}$ & $\mathrm{t}$ & $(0.500,0.496)$ & $(0.500,0.470)$ & $(0.500,0.479)$ & $(0.500,0.539)$ & $(0.497,0.487)$ & $(0.504,0.499)$ \\
\hline & $\mathrm{SF3}$ & $\gamma_{\mathrm{sf}}$ & 203 & 634 & 511 & 1421 & 169 & 54 \\
\hline \multirow{3}{*}{ Pyr. II } & \multirow{3}{*}{$\mathrm{SF} 1$} & $\mathrm{t}$ & $(0.000,0.480)$ & $(0.000,0.446)$ & $(0.000,0.455)$ & $(0.000,0.434)$ & $(0.000,0.535)$ & $(0.000,0.541)$ \\
\hline & & & 165 & 321 & 282 & 1022 & 119 & 47 \\
\hline & & $\gamma_{\mathrm{sf}}$ & $236^{\circ}$ & & & & & \\
\hline
\end{tabular}

${ }^{a}$ Ref. [36], DFT, SIESTA, GGA-PBE, Norm-conserving.

${ }^{\mathrm{b}}$ Ref. [23], DFT, VASP, GGA-PW91, US.

${ }^{\mathrm{c}}$ Ref. [37], DFT, VASP, GGA-PW91, US.

${ }^{\mathrm{d}}$ Ref. [38], DFT, VASP, GGA-PW91, PAW.

${ }^{\mathrm{e}}$ Ref. [39], DFT, VASP, [S]GGA-PW91, PAW.

${ }^{\mathrm{f}}$ Ref. [40], DFT, VASP, GGA-PBE, PAW.

${ }^{\mathrm{g}}$ Ref. [41], DFT, VASP, GGA-PW91, US.

${ }^{\mathrm{h}}$ Ref. [14], DFT, VASP, GGA-PW91, PAW.

${ }^{i}$ Ref. [13], DFT, VASP, GGA-PBE, PAW.

${ }^{j}$ Ref. [42], DFT, VASP, GGA-PW91, US.

${ }^{\mathrm{k}}$ Ref. [43], DFT, VASP, GGA-PBE, PAW.

${ }^{1}$ Ref. [15], DFT, PWSCF, GGA-PBE, US.

${ }^{m}$ Ref. [18], DFT, PWSCF, GGA-PBE, US.

${ }^{\mathrm{n}}$ Ref. [17], DFT, PWSCF, GGA-PBE, US.

${ }^{\circ}$ Ref. [44], DFT, VASP, GGA-PW91, US. 
On the Pyr. I plane, all metals show three stable SFs. One is relevant to $\langle\mathbf{a}\rangle$ slip on the narrow plane and two are relevant to $\langle\mathbf{c}+\mathbf{a}\rangle$ slip on the wide plane. The position of the SF varies among different metals and cannot be determined solely by crystal symmetry. Specially, in $\mathrm{Zn}$ and $\mathrm{Cd}$, where the $c / a$ ratio $(\sim 1.9)$ is very far from the ideal hcp value (1.633), SF3 has a much lower energy than SF2, while in the other metals SF2 has a lower energy than SF3. On the Pyr. II plane, all metals show a single stable SF that is relevant to $\langle\mathbf{c}+\mathbf{a}\rangle$ dissociation. The positions of the SFs again vary among the metals, but all are along the $\langle\mathbf{c}+\mathbf{a}\rangle$ direction. Our results on the Pyr. I plane are also in good agreement with a few recently available DFT calculations using full relaxation.

Overall, the existence and approximate positions of the stable SFs found in the DFT calculations are consistent with the expectations based on crystal symmetry. The position of these stable SFs are also in qualitative agreement with some full $\gamma$-surface calculation using the standard method [23]. Therefore, we expect these are likely to be all of the stable SFs on the five slip planes examined in this study.

Figure 5 shows the SF structure and the atomic displacements on one side of the SF plane. Figure 6 shows the atomic displacements as a function of atomic layer away from the slip plane (in $x_{3}$ direction). Here, we focus on $\mathrm{Mg}$, $\mathrm{Ti}$ and $\mathrm{Zr}$, which are the important hcp structural metals. On the Basal plane, the in-plane displacements are negligible while small out-of-plane displacements extending to 2-3 atomic layers are seen in $\mathrm{Ti}$ and $\mathrm{Zr}$, as shown in Fig. 5a and Fig. 6a. On the Prism I plane, the in-plane displacements are negligible but the out-of-plane displacements are substantial over many atomic layers for both SF1 and SF2 (see Fig. 5b, $\mathrm{f}$ and $\mathrm{k}$, and Fig $6 \mathrm{~b}$ and c). This is consistent with the fact that full relaxation does not affect the SF energy on the Basal and Prism I planes. Furthermore, the direction of the out-of-plane displacement alternates between atom layers, indicating some atomic shuffling process along the slip path. In contrast, for the SF1 on the Prism II plane, significant out-of-plane displacements are seen in Ti and $\mathrm{Zr}$ and significant in-plane displacements are seen over many atomic layers in all three metals (see Fig. 5c, g and 1, and Fig. 6d). This explains the large differences in SF energy calculated using full atom relaxation and the standard approach (out-of-plane relaxation only) [23].

On the Pyr. I and II planes, all SFs have both in-plane and out-of-plane displacements extending 3-6 atomic layers into the surrounding material, as shown in Fig. $5 \mathrm{~d}$ and e for $\mathrm{Mg}, \mathrm{h}, \mathrm{i}$ and $\mathrm{j}$ for $\mathrm{Ti}, \mathrm{m}, \mathrm{n}$ and $\mathrm{o}$ for $\mathrm{Zr}$. The corresponding displacement plots are shown in Fig. 6e-h. The SF1 on the Pyr. I plane, as shown in Fig. 5 h, m and Fig. 6e, shows substantial out-of-plane displacements and in-plane displacements perpendicular to the slip direction. This SF is close to a two-layer 

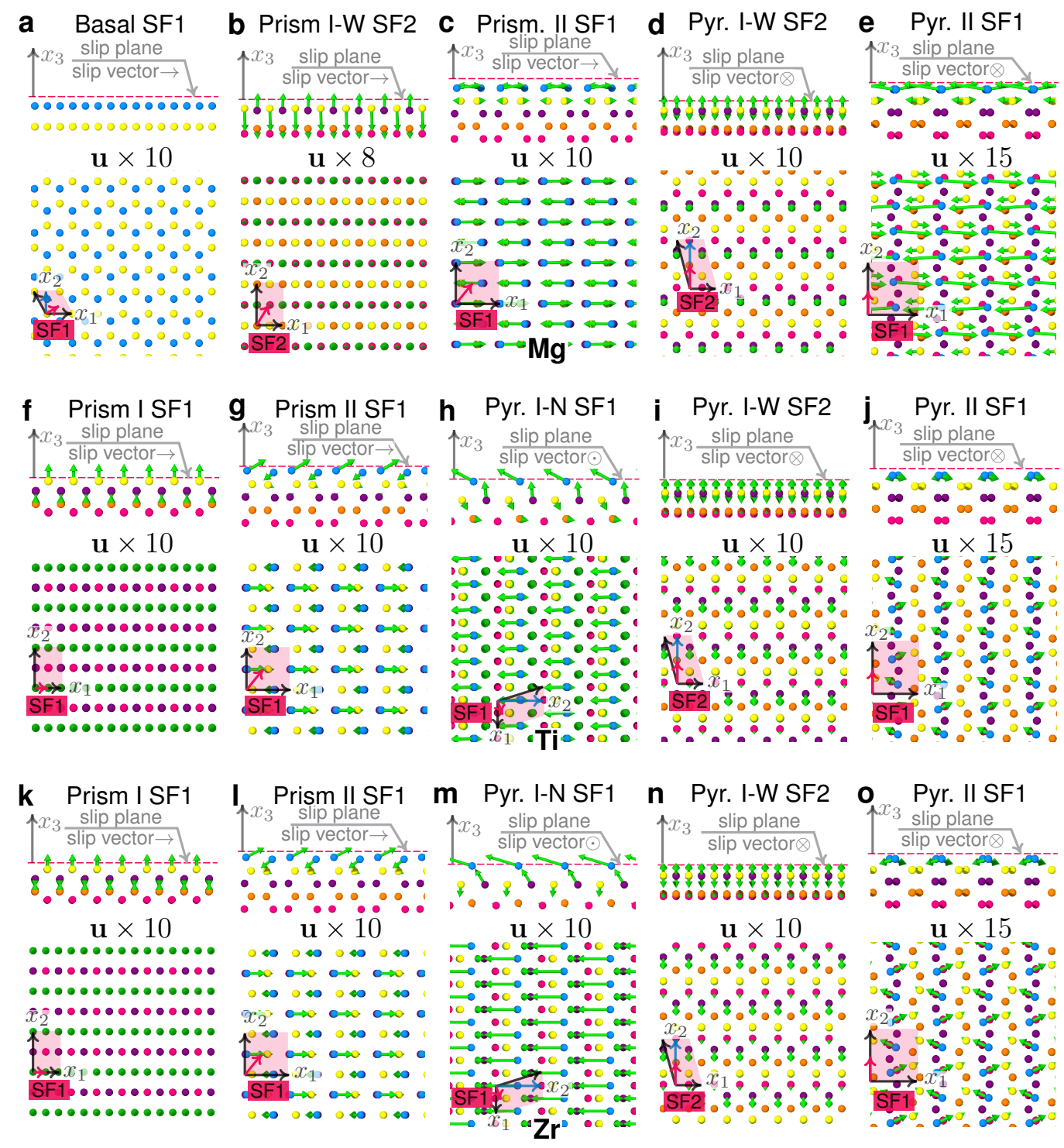

Figure 5: Atomic structure and displacements on one side of the stacking fault plane. (a-e) $\mathrm{Mg}$, (f-j) Ti, (k-o) Zr. In each subpanel, the upper and lower pictures show structure and displacement viewed in the direction of the slip plane and perpendicular to the slip plane, respectively. The green arrows show the atomic displacements magnified by the respective value $(\mathbf{u} \times)$ shown in each figure. 

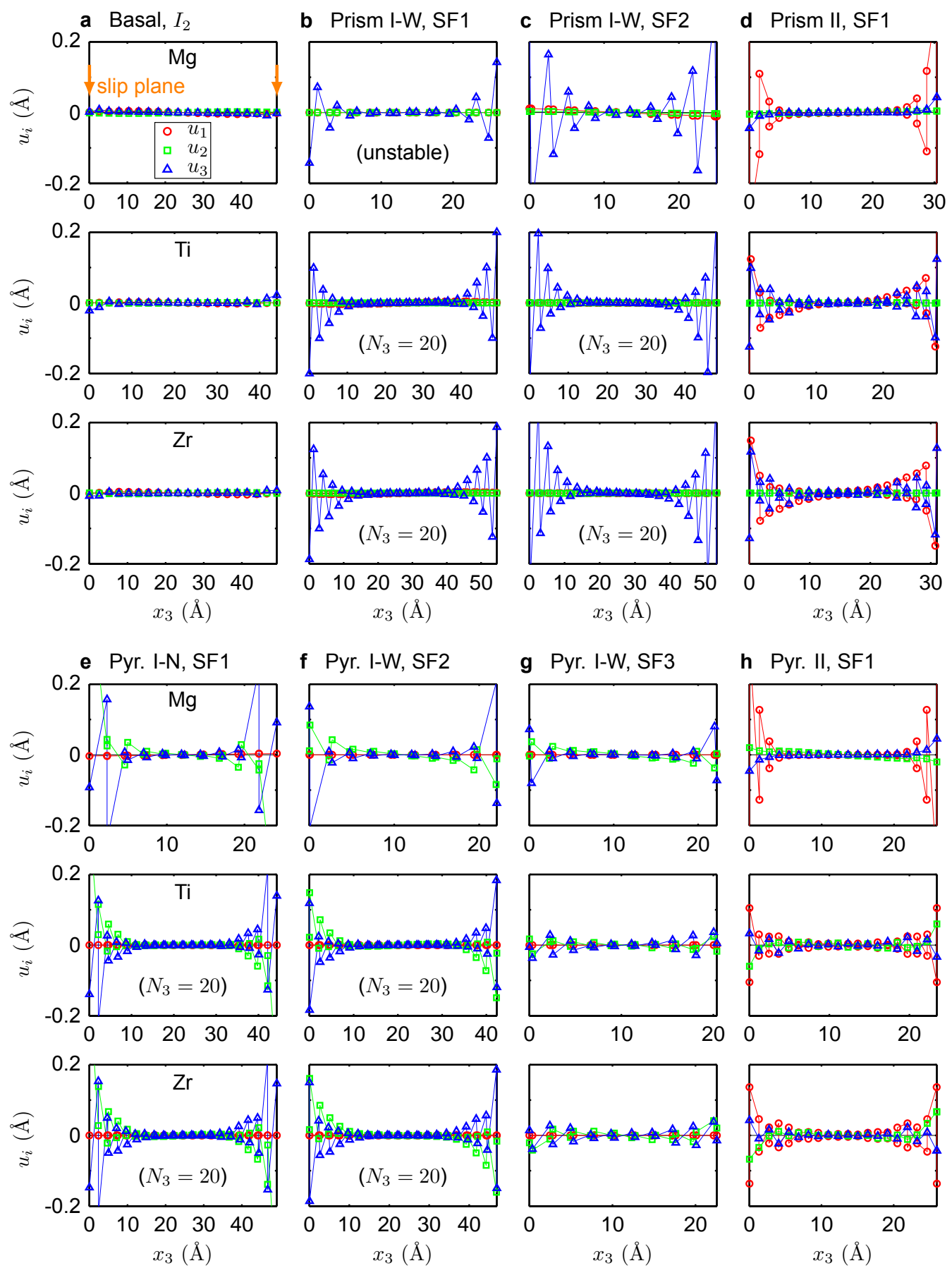

Figure 6: Atomic displacements at various SFs. $u_{1}$ and $u_{2}$ are the displacement in the slip plane, $u_{3}$ is out-of-plane displacement perpendicular to the slip plane (see Fig. 5 for the respective orientation of the coordinate system). Unless otherwise noted, calculations are performed with $N_{3}=10$. 
twin on the Pyr. I plane $[45,16]$. However, the DFT-calculated SF position shows some deviation from the twin vector based on crystal symmetry $[45,16,17]$, presumably since this structure is a nascent two-layer twin and not a full twin. For the SF2 and SF3 on Pyr. I, the in-plane displacements are all in the slip direction, while SF1 on the Pyr. II plane has in-plane displacements in both the slip and perpendicular-to-slip directions. Overall, the directions of the displacements are similar, but the magnitudes vary among the three different metals. For example, the in-plane displacements of SF2 on the Pyr. I plane are small in Mg as compared to $\mathrm{Ti}$ and $\mathrm{Zr}$, while the opposite is true for SF1 on the Pyr. II planes. In addition, the in-plane displacements are relatively small with respective to the slip vector in the slip direction and decay quickly from the slip plane. This suggests that the slip is localized on the SF plane. The clear existence of the SF with well-defined structure suggests that dislocation dissociation into zonal dislocations is unlikely. On the Pyr. II plane, the direction of the in-plane displacement perpendicular to the slip direction alternates between atom rows on each atomic plane, as expected from examination of the hard-sphere model. This suggests atomic shuffling along the slip path of the $\langle\mathbf{c}+\mathbf{a}\rangle$ Burgers vector on the Pyr. II plane.

Overall, the stable SF structures on all planes except the Basal have substantial out-of-plane atomic displacements and some have additional in-plane atomic displacements. These displacements are the minimum required for slip on the respective planes and additional atomic displacements are expected along the slip path. This suggests slip on these planes as mediated by dislocations will likely have a relatively high Peierls stress and low mobility. At low resolved shear stresses, slip will also involve some thermal activations, thus being temperature-dependent and more difficult than slip on Basal planes in materials where the Basal $\langle\mathbf{a}\rangle$ is stable. The out-of-plane displacements and in-plane displacements perpendicular to the slip direction also suggests that applied non-Schmid stresses will also have some effects on dislocation slip behavior, such as the Peierls stress.

The varying degree of in-plane displacements on the Basal, Prism and Pyramidal planes should have different effects on the accuracy of the local energy minimum, i.e. stable SF energy, calculated by the standard method. Figure 7 shows the $\gamma$-line calculated by the standard method and the single-point stable SF determined using full atomic relaxation. On the Basal and Prism I planes, both the position and energy of the stable SF are identical in both methods, as expected from the absence of in-plane atomic displacements on these two planes. However, on the Pyr. I and II planes, both the position and energy of SF differ, with the effects of full atomic relaxation varying among the different metals. On the Pyr. I plane, the differences between the two methods are small in $\mathrm{Mg}$ but 

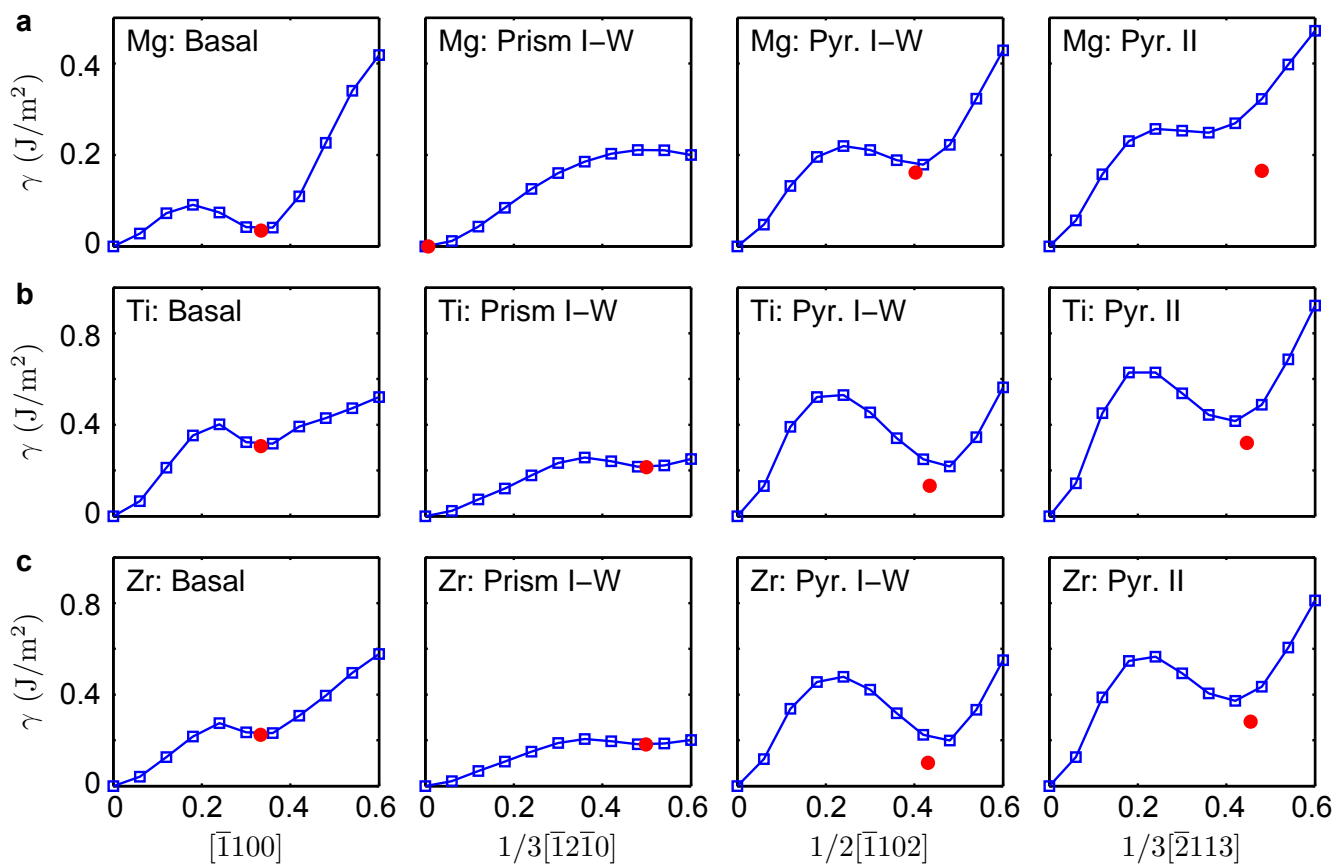

Figure 7: DFT-calculated $\gamma$-line of (a) $\mathrm{Mg}$, (b) Ti and (c) $\mathrm{Zr}$ using the standard method (out-ofplane relaxation only), along with the fully-relaxed stacking fault energy (filled dot). No stable stacking fault is found at $\langle\mathbf{a}\rangle / 2$ on the Prism plane in $\mathrm{Mg}$. 
substantial ( $\sim 5 \%)$ in Ti and Zr. On the Pyr. II plane, the position and energies are substantially different $(\sim 35 \%)$ in $\mathrm{Mg}$ and moderately different $\mathrm{Ti}$ and $\mathrm{Zr}$. These results are fully consistent with the relative magnitude of the in-plane displacements shown in Fig. 5 and Fig. 6. For Mg, use of the standard $\gamma$-line would suggest that Pyr. I $\langle\mathbf{c}+\mathbf{a}\rangle$ might be more stable than Pyr. II $\langle\mathbf{c}+\mathbf{a}\rangle$, which is not supported by the full results for which the two SF energies are very similar. In $\mathrm{Ti}$ and $\mathrm{Zr}$, conclusions about relative stability of Pyr. I and Pyr. II are not different from those drawn from the full results, although the actual energy differences, and the partial dislocation dissociation distances, would not be determined accurately when using the energies from the standard approach. Furthermore, on the Pyramidal planes, the entire $\gamma$-line calculated using the standard approach is not accurate in predicting properties such as dislocation nucleation and material theoretical shear strength. New approaches, as discussed below, should be used to obtain more accurate $\gamma$-lines in future studies.

\section{Implications for dislocation structures and slip in hcp metals}

\section{1. $\langle\mathbf{c}+\mathbf{a}\rangle$ dissociation on the Pyr. I plane}

The current work and a previous study [23] show that there exist two SFs associated with $\langle\mathbf{c}+\mathbf{a}\rangle$ slip on the Pyr. I plane. The corresponding $\langle\mathbf{c}+\mathbf{a}\rangle$ dislocations can thus have two different dissociations, one into two partials and the other into three partials, as shown in Fig. 8a and b. The energetically favorable one is of practical importance. However, energetic comparisons based only on elasticity cannot reliably determine the energetically favorable dissociation because one case has two partial dislocation cores while the other has three [4]. Nevertheless, for most metals examined in this study, SF2 and SF3 on the Pyr. I plane have quite different energies. We thus expect that, in the three-dislocationdissociation case, one of the dislocation separations, either $r_{12}$ or $r_{23}$, will be quite small. We therefore compute the equilibrium partial separation for the possible three-dislocation-dissociation case, and draw general conclusions based on the calculated partial separation. The stacking fault energy and partial Burgers vector (stacking fault vector) shown in Table. 3 combine to determine the equilibrium partial dislocation dissociation distance within anisotropic linear elasticity theory. For glide dissociation of various dislocations, the interaction force between two dislocations $\mathbf{b}_{i}$ and $\mathbf{b}_{j}$ in the radial direction is $f_{i j}=K_{i j} / r_{i j}$, where $K_{i j}$ is a interaction energy prefactor determined by the elastic constants and the Burgers vectors of the two partial dislocations (see, for example, Ref $[4,46]$ ). This inter-

action force, if repulsive, is balanced by the attractive configurational SF force $\gamma_{\mathrm{sf}}$ 
and an equilibrium partial separation distance is established. Using such a force balance argument, we can determine all the equilibrium partial separations. For the case of three partial dislocations, the Burgers vectors are $\mathbf{b}_{1}, \mathbf{b}_{2}$ and $\mathbf{b}_{3}$ and partial separations are $r_{12}$ and $r_{23}$, as shown in Fig 8b. At equilibrium, the total radial force on $b_{1}$ is

$$
f_{\mathbf{b}_{1}}=f_{12}+f_{13}-f_{S F 2}=-\frac{K_{12}}{r_{12}}-\frac{K_{13}}{r_{12}+r_{23}}+\gamma_{S F 2}=0
$$

where $f_{i j}$ is the elastic interaction force between dislocation pair $\mathbf{b}_{i}$ and $\mathbf{b}_{j} \cdot f_{S F 2}$ is the configurational force due to the stacking fault SF2 between dislocation $\mathbf{b}_{1}$ and $\mathbf{b}_{2}$, which is simply the stacking fault energy density $\gamma_{S F 2}$. Similarly, the total radial force on $b_{3}$ is

$$
f_{\mathbf{b}_{3}}=f_{13}+f_{23}-f_{S F 3}=\frac{K_{13}}{r_{12}+r_{23}}+\frac{K_{23}}{r_{23}}-\gamma_{S F 3}=0
$$

In the above Eqs. (2) and (3), the only unknowns are $r_{12}$ and $r_{23}$, which can be found by solving the pair of equations simultaneously.
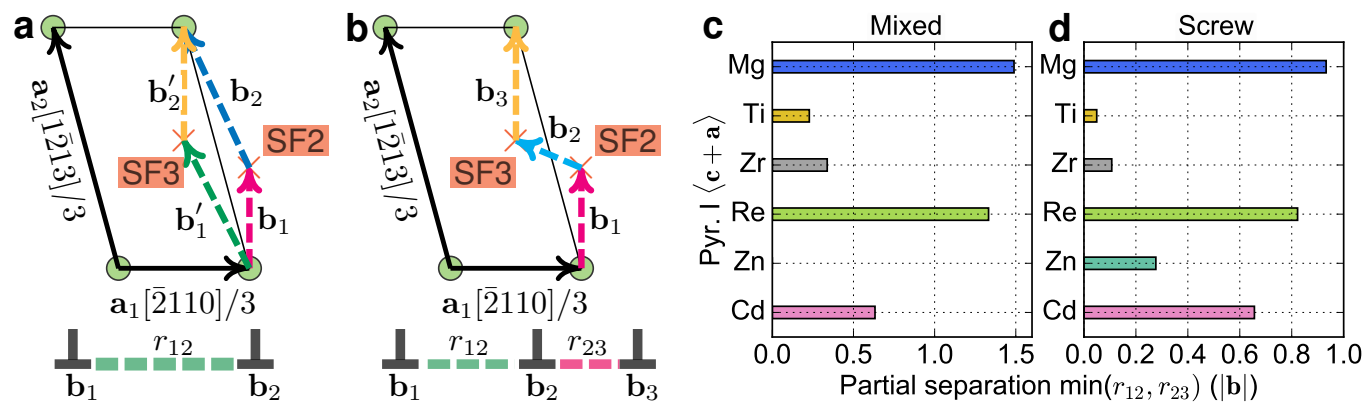

Figure 8: Schematic and partial separation of $\langle\mathbf{c}+\mathbf{a}\rangle$ dislocation dissociations on the Pyr. I plane. (a) Dissociation into two partials. (b) Dissociation into three partials. (c,d) Smaller of the two possible partial separations, $\min \left(r_{12}, r_{23}\right)$, normalized by the $\langle\mathbf{c}+\mathbf{a}\rangle$ Burgers vector for the dissociation into three partials for the mixed and screw $\langle\mathbf{c}+\mathbf{a}\rangle$ dislocation.

Figure $8 \mathrm{c}$ and $\mathrm{d}$ show the smaller of the two equilibrium partial separations for the three partial dissociation case, using the experimental material elastic constants and lattice parameters shown in Table 2. For all metals and both the mixed and screw $\langle\mathbf{c}+\mathbf{a}\rangle$ dislocations, the smallest separation is quite small. For $\mathrm{Ti}, \mathrm{Zr}$ and $\mathrm{Zn}$, the smallest separation is less than the $a$ lattice parameter. For $\mathrm{Mg}$, which has the largest of the smaller separations among all the 6 metals, recent direct DFT 
simulations show that the $\langle\mathbf{c}+\mathbf{a}\rangle$ dissociates into only two partials on the Pyr. I plane [19]. Therefore, dissociation into three partials is highly unlikely in these metals and dissociation into two partials as shown in Fig. 8a is expected.

\section{2. $\langle\mathbf{c}\rangle$ dissociation on the Prism planes}

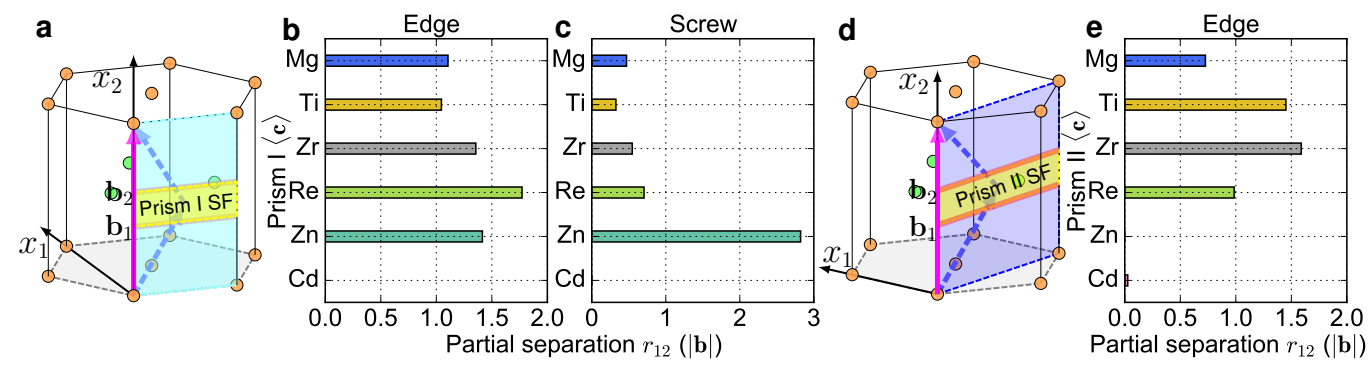

Figure 9: Schematic and partial separation of $\langle\mathbf{c}\rangle$ dislocation dissociations on the Prism planes. (a) Dissociation on the Prism I plane. (b,c) The equilibrium partial separation $r_{12}$ normalized by the Burgers vector $\langle\mathbf{c}\rangle$ for the edge and screw $\langle\mathbf{c}\rangle$ dislocation on the Prism I plane. The dissociation distance for $\mathrm{Cd}$ is not computed as the corresponding SF is not stable in the current DFT calculations. (d) Dissociation on the Prism II plane. (e) The equilibrium partial separation $r_{12}$ normalized by the Burgers vector $\langle\mathbf{c}\rangle$ for the edge $\langle\mathbf{c}\rangle$ dislocation on the Prism II plane. Dissociation of the screw $\langle\mathbf{c}\rangle$ on the Prism II plane is not expected $\left(r_{12}<0\right)$.

The $\langle\mathbf{c}\rangle$ dislocations can glide-dissociate onto the Prism I and II planes or climb-dissociate onto the Basal plane with a Basal extrinsic SF [5, 47]. An energetic comparison between the various dissociation configurations for the $\langle\mathbf{c}\rangle$ is again intricate, because the partial Burgers vectors, and thus their core energies, change substantially. However, the SF energy (except Cd on the Prism I plane) is quite high on the Prism planes, suggesting very narrow partial separations. Figure 9 shows the equilibrium partial separations for glide-dissociation on the Prism planes as calculated by anisotropic elasticity. For all cases except the screw $\langle\mathbf{c}\rangle$ in $\mathrm{Zn}$, the partial separation on the Prism plane is less than or comparable to the Burgers vector $\langle\mathbf{c}\rangle$. This suggests that climb-dissociation onto the Basal plane is quite feasible for the edge $\langle\mathbf{c}\rangle$, as driven by the low SF energy on the Basal plane and by the strong angular preference of $90^{\circ}$ for partials with similar edge characters [4]. This is also consistent with recent MD simulations showing spontaneous $\langle c\rangle$ basal-climb-dissociation in $\mathrm{Mg}$ [47] and various experimental observations showing long, straight $\langle\mathbf{c}\rangle$ dislocations on the Basal planes $[48,49,50]$. The screw $\langle\mathbf{c}\rangle$ partial dislocations have a very narrow core separation $(\mathrm{r}<|\mathbf{b}|$ except 
$\mathrm{Zn}$ ) on the Prism I plane and so no dissociation is expected on the Prism II planes. This suggests that the screw $\langle\mathbf{c}\rangle$ dislocations have compact cores and, if they exist, their cross-slip may be relatively easy.

\subsection{Equilibrium partial separations for $\langle\mathbf{a}\rangle$ and $\langle\mathbf{c}+\mathbf{a}\rangle$ dislocations}

Figure 10 shows the equilibrium separation distance for the $\langle\mathbf{a}\rangle$ and $\langle\mathbf{c}+\mathbf{a}\rangle$ dislocations, as calculated by anisotropic linear elastic theory using experimental materials properties in Table 2 and the DFT-computed SF information in Table 3. Full energetic comparison among the different dissociation configurations have been presented in Ref. [4]. Here, we focus on the broad implications from the calculated equilibrium separation and comparison with recent available direct DFT calculations. For the $\langle\mathbf{a}\rangle$ dislocation, dissociation into Shockley (mixed edge/screw) partials is expected on the Basal plane in all metals. In contrast, the SF position on the Prism I plane suggests that $\langle\mathbf{a}\rangle$ edge and screw should dissociate into pure edge and pure screw partials, respectively. Similarly, the SF position on the Pyr. I plane suggests $\langle\mathbf{a}\rangle$ edge and screw dissociation into near-pure edge and near-pure screw, respectively. $\mathrm{In} \mathrm{Mg}, \mathrm{Re}, \mathrm{Zn}$ and $\mathrm{Cd}$, where the Basal $I_{2}$ energy is substantially lower than the Prism I SF1, the $\langle\mathbf{a}\rangle$ dissociation distance is much larger on the Basal plane than on Prism I or Pyr. I plane. The screw $\langle\mathbf{a}\rangle$ has a dissociation distance of at least $3 a$ on Basal plane, and slightly smaller dissociation distances on the Prism and Pyr. I planes. The wider dissociation distance of the screw $\langle\mathbf{a}\rangle$ suggests that this dislocation is rather stable on the Basal planes, so that cross-slip via partial constriction is difficult in these metals. In contrast, $\mathrm{Ti}$ and $\mathrm{Zr}$ have Basal screw $\langle\mathbf{a}\rangle$ dissociation distances close to $a$, suggesting that the screw $\langle\mathbf{a}\rangle$ dissociated on the Basal plane (if stable) can easily cross-slip to the energetically favorable Prism plane [4]. These conclusions are all consistent with the observed dominance of $\langle\mathbf{a}\rangle$ slip in the different metals [51]. For the $\langle\mathbf{c}+\mathbf{a}\rangle$ dislocations on both Pyr. I and II planes, all metals show fairly large dissociation distances. In particular, the dissociation distances are very large for Pyr. I $\langle\mathbf{c}+\mathbf{a}\rangle$ in $\mathrm{Ti}, \mathrm{Zr}$ and $\mathrm{Cd}$ and $\mathrm{Pyr}$. II $\langle\mathbf{c}+\mathbf{a}\rangle$ in $\mathrm{Zn}$ and $\mathrm{Cd}$. The large dissociation distances pose a computational challenge for DFT simulations of full dislocations. Finally, comparison with available DFT-based atomic simulations, as shown in Fig. 10 a, $\mathrm{b}, \mathrm{d}, \mathrm{f}, \mathrm{h}, \mathrm{i}, \mathrm{j}$, shows good matches with the elasticity calculations here.

\section{Discussion and conclusions}

Our DFT calculations show that SF3 on the Pyr. I plane is stable in all 6 metals, while the $\gamma$-surface by DFT in $\operatorname{Zr~[17]~and~} \gamma$-surface by MEAM in Ti [52] 

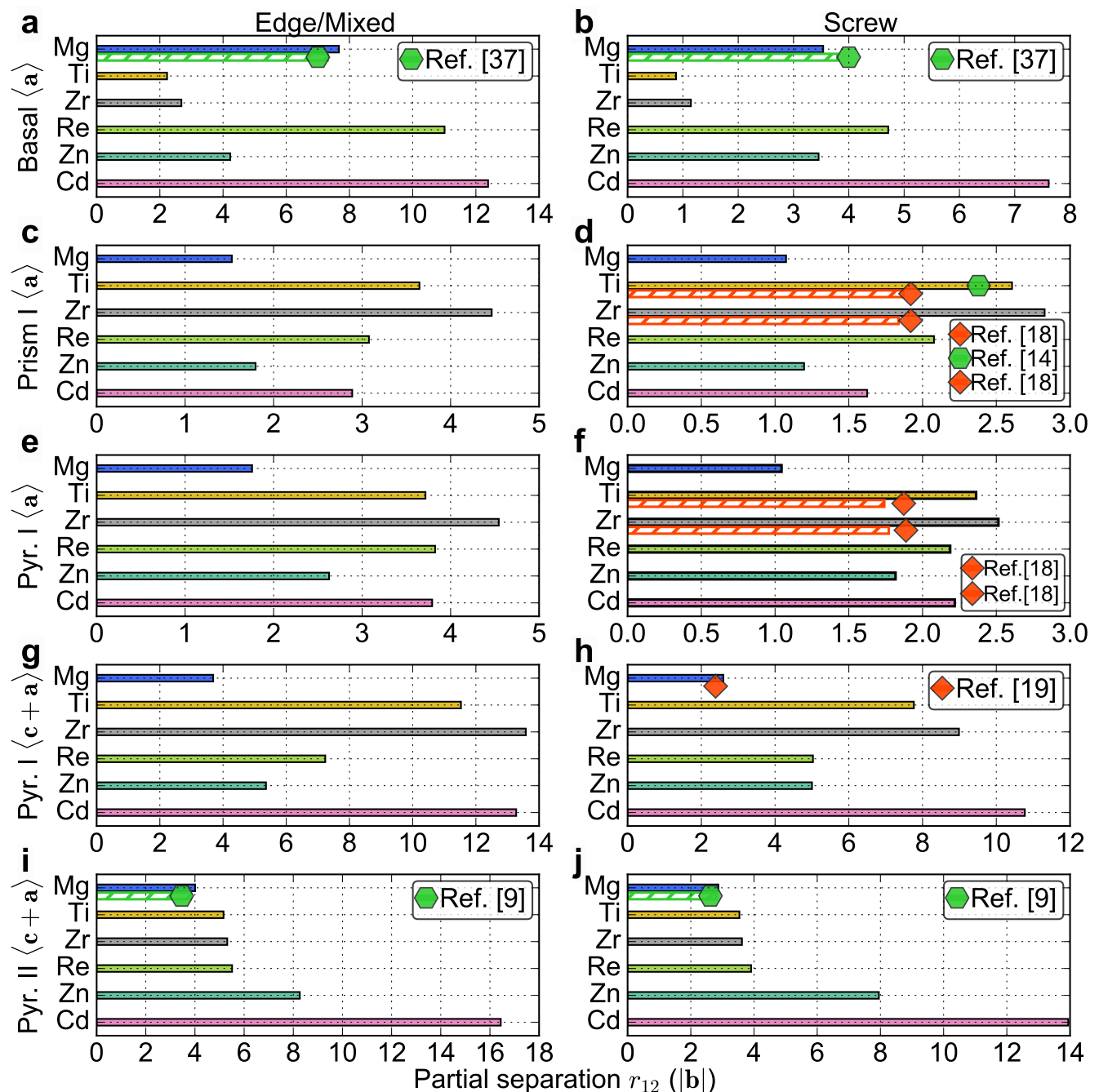

Figure 10: Equilibrium partial dislocation separation $r_{12}$ normalized by the respective Burgers vector $(\langle\mathbf{a}\rangle$ or $\langle\mathbf{c}+\mathbf{a}\rangle)$ as calculated by anisotropic linear elastic theory. Filled-bars: lattice and elastic constants from experiments (Table 2) and stacking fault energies from DFT (Table 3); Hatched bars: all properties from DFT calculations; Symbols: full DFT simulations of dislocation cores using flexible boundary conditions (hexagon) and periodic boundary conditions (diamond). 
seem to suggest that SF3 is not stable along the $\mathbf{e}_{2}$ direction. Additional DFT calculations in Ti and Zr on slightly perturbed SF structures (1-3\% in the $\pm \mathbf{e}_{1}$ and $\pm \mathbf{e}_{2}$ directions) from the relaxed SF3 position show that the perturbed SFs relax back to SF3. Stacking faults with larger perturbations from SF3 $(>3 \% a)$ relax into SF2. This suggests SF3 is marginally stable close to the true fault position and very likely a shallow local minimum not resolved in the $\gamma$-surface calculated using the standard method. Additional checks with different supercell sizes $\left(N_{1}\right.$, $N_{2}$ and $N_{3}$ ) yield the same conclusion. In all metals except $\mathrm{Mg}$, the energy of SF3 is substantially different from that of SF2, suggesting that the high energy $\mathrm{SF}$ is very unlikely to form through dislocation dissociation, as demonstrated in Section 5.1. In $\mathrm{Mg}$, direct DFT calculation [19] on the $\langle\mathbf{c}+\mathbf{a}\rangle$ dislocation core structure on the Pyr. I plane shows that the $\langle\mathbf{c}+\mathbf{a}\rangle$ dislocation dissociates into two partials. All these results suggest that the high energy stacking fault (SF3 in $\mathrm{Mg}$, Ti, $\mathrm{Zr}$, and $\mathrm{Re}, \mathrm{SF} 2$ in $\mathrm{Zn}$ and $\mathrm{Cd}$ ) is not relevant to dislocation dissociation and its physical properties.

Since the $\gamma$-line profile determines a set of important material properties, its accurate measurement is of fundamental importance. The current DFT results shown in Fig. 5 and 6 indicate full relaxation is essential on Prism II, Pyr. I and II planes in all hcp metals examined here. The procedure detailed in Section 3 is only applicable for determining the stable SF. For any other unstable point on the $\gamma$-line or $\gamma$-surface, additional constraints have to be applied to determine the SF energy. In calculations of pure metals, the $\gamma$-line can be obtained through the nudged elastic band (NEB) method calculations, as demonstrated in a recent work by Kwasniak et al. [13]. Alternatively, the procedure proposed by Morris et al. [10] may be used to calculate the $\gamma$-line or even the full $\gamma$-surface. Therefore, the procedures to obtain accurate SF information in pure metals are readily available. Accurate $\gamma_{\text {sf }}$ and $\gamma_{\text {usf }}$ thus obtained can be used to better predict dislocation dissociation [4] and crack-tip behavior in hep metals [53]. In SF calculations of metals with solid solution alloying, the NEB method can be applied to obtain a $\gamma$-line, but the result depends on the choice of the supercell size $N_{1}$ and $N_{2}$, in addition to the usual $N_{3}$-dependence, and will depend on how the solutes are distributed. The physical interpretation of such computed $\gamma$-lines is intricate. The calculations of the full $\gamma$-surface with full relaxation are not well-defined in systems with solid solution, nor is there any clear understanding on its relation to the mechanical properties of alloys with solutes.

In summary, we conducted a comprehensive study on all the stable stacking fault on 5 slip planes for 6 hcp metals using crystal symmetry and DFT calculations. The atomic structures of the SFs are similar among all the metals examined 
here. Crystal symmetry dictates the overall in-plane and out-of-plane displacements, while the details of atomic bonding determine the SF energies and affect the precise positions and atomic relaxations in a quantitative manner. This suggests that plastic slip share many common features in the family of hcp metals. Differences in plasticity and the contribution of each slip system toward total plastic strain are due to the activation of different slip systems, which depends on the Peierls stress and the stability of the dislocation core of the respective slip systems. Special cases considered here suggest that $\langle\mathbf{c}+\mathbf{a}\rangle$ dislocation dissociation into three partials on the Pyr. I plane is highly unlikely and that $\langle\mathbf{c}\rangle$ dislocation dissociation on the Prism planes is unlikely to be stable against basal-climb dissociation in these hcp metals. While the above conclusions are general, robust and not strongly influenced by dislocation core effects, we shall still emphasize that the current framework does not provide the full scope of dislocation properties, such as the dislocation core structure. Since dislocation core structures dictate dislocation behavior in response to applied load, obtaining the true dislocation core structures and properties, either through DFT calculations or experimental methods, is still essential. The present comprehensive set of results on the energy and structure of hcp stacking fault thus provides a useful benchmark for further theoretical computations, for guiding future direct atomistic simulations, and for the development of accurate empirical interatomic potentials to enable more complex simulations of dislocation phenomena.

\section{Acknowledgments}

The authors acknowledge financial support of this work through a grant from the Swiss National Science Foundation entitled "Control of Atomistic Mechanisms of Flow in Magnesium Alloys to Achieve High Ductility" (project \#162350), as well as computer time supported by a grant from the Swiss National Supercomputing Centre (CSCS) under project ID s631.

\section{References}

\section{References}

[1] V. Vitek, Intrinsic stacking faults in body-centred cubic crystals, Philosophical Magazine 18 (1968) 773-786.

[2] V. Vitek, Structure of dislocation cores in metallic materials and its impact on their plastic behaviour, Progress in Materials Science 36 (1992) 1-27. 
[3] J. R. Rice, Dislocation nucleation from a crack tip: An analysis based on the peierls concept, Journal of the Mechanics and Physics of Solids 40 (1992) 239-271.

[4] Z. Wu, B. Yin, W. A. Curtin, Energetics of dislocation transformations in hcp metals, Acta Materialia 119 (2016) 203-217.

[5] J. P. Hirth, J. Lothe, Theory of Dislocations, 2 ed., John Wiley \& Sons, 1982.

[6] R. Meyer, L. J. Lewis, Stacking-fault energies for $\mathrm{Ag}, \mathrm{Cu}$, and $\mathrm{Ni}$ from empirical tight-binding potentials, Physical Review B 66 (2002) 052106-4.

[7] Y. Minonishi, S. Ishioka, M. Koiwa, S. Morozumi, M. Yamaguchi, The core structures of a $1 / 3\langle\overline{1} \overline{1} 23\rangle\{11 \overline{2} 2\}$ edge dislocation under applied shear stresses in an h.c.p. model crystal, Philosophical Magazine A 45 (1982) $835-850$.

[8] D. J. Bacon, M. H. Liang, Computer simulation of dislocation cores in h.c.p. metals I. interatomic potentials and stacking-fault stability, Philosophical Magazine A 53 (1986) 163-179.

[9] M. Ghazisaeidi, L. G. Hector Jr, W. A. Curtin, First-principles core structures of $\langle c+a\rangle$ edge and screw dislocations in Mg, Scripta Materialia 75 (2014) 42-45.

[10] J. R. Morris, J. Scharff, K. M. Ho, D. E. Turner, Y. Y. Ye, M. H. Yoo, Prediction of a $\{11 \overline{2} 2\}$ hcp stacking fault using a modified generalized stackingfault calculation, Philosophical Magazine A 76 (1997) 1065-1077.

[11] Y. Dou, J. Zhang, Effects of structural relaxation on the generalized stacking fault energies of hexagonal-close-packed system from first-principles calculations, Computational Materials Science 98 (2015) 405-409.

[12] P. Kwaśniak, P. Śpiewak, H. Garbacz, K. J. Kurzydłowski, Plasticity of hexagonal systems: Split slip modes and inverse peierls relation in $\alpha$-Ti, Physical Review B 89 (2014) 144105-6.

[13] P. Kwasniak, H. Garbacz, K. J. Kurzydlowski, Solid solution strengthening of hexagonal titanium alloys: Restoring forces and stacking faults calculated from first principles, Acta Materialia 102 (2016) 304-314. 
[14] M. Ghazisaeidi, D. R. Trinkle, Core structure of a screw dislocation in Ti from density functional theory and classical potentials, Acta Materialia 60 (2012) 1287-1292.

[15] E. Clouet, Screw dislocation in zirconium: An ab initio study, Physical Review B 86 (2012) 144104-11.

[16] N. Chaari, E. Clouet, D. Rodney, First-principles study of secondary slip in zirconium, Physical Review Letters 112 (2014) 075504-.

[17] N. Chaari, E. Clouet, D. Rodney, First order pyramidal slip of $1 / 3<1 \overline{2} 10>$ screw dislocations in zirconium, Metallurgical and Materials Transactions A 45 (2014) 5898-5905.

[18] E. Clouet, D. Caillard, N. Chaari, F. Onimus, D. Rodney, Dislocation locking versus easy glide in titanium and zirconium, Nature Materials 14 (2015) 931-936.

[19] M. Itakura, H. Kaburaki, M. Yamaguchi, T. Tsuru, Novel cross-slip mechanism of pyramidal screw dislocations in magnesium, Physical Review Letters 116 (2016) 225501-5.

[20] M. L. Kronberg, Atom movements and dislocation structures for plastic slip in single crystals of $\beta$-uranium, Journal of Nuclear Materials 1 (1959) 85-95.

[21] M. L. Kronberg, Atom movements and dislocation structures in some common crystals, Acta Metallurgica 9 (1961) 970-972.

[22] I. P. Jones, W. B. Hutchinson, Stress-state dependence of slip in Titanium6Al-4V and other H.C.P. metals, Acta Metallurgica 29 (1981) 951-968.

[23] A. Poty, J.-M. Raulot, H. Xu, J. Bai, C. Schuman, J.-S. Lecomte, M.-J. Philippe, C. Esling, Classification of the critical resolved shear stress in the hexagonal-close-packed materials by atomic simulation: Application to $\alpha$ zirconium and $\alpha$-titanium, Journal of Applied Physics 110 (2011) 01490515.

[24] G. Kresse, J. Furthmüller, Efficient iterative schemes for ab initio totalenergy calculations using a plane-wave basis set, Physical Review B 54 (1996) 11169-11186. 
[25] G. Kresse, D. Joubert, From ultrasoft pseudopotentials to the projector augmented-wave method, Physical Review B 59 (1999) 1758-1775.

[26] J. P. Perdew, K. Burke, M. Ernzerhof, Generalized gradient approximation made simple, Physical Review Letters 77 (1996) 3865-3868.

[27] P. E. Blöchl, Projector augmented-wave method, Physical Review B 50 (1994) 17953-17979.

[28] M. Methfessel, A. T. Paxton, High-precision sampling for Brillouin-zone integration in metals, Physical Review B 40 (1989) 3616-3621.

[29] H. J. Monkhorst, J. D. Pack, Special points for brillouin-zone integrations, Physical Review B 13 (1976) 5188-5192.

[30] C. S. Barrett, T. B. Massalski, Structure of Metals, International Series on Materials Science and Technology, 3 ed., Pergamon, 1980.

[31] G. Simmons, H. Wang, Single Crystal Elastic Constants and Calculated Aggregate Properties. A Handbook, 2 ed., The MIT Press, 1971.

[32] J. Friedel, Metallic alloys, Il Nuovo Cimento 7 (1958) 287-311.

[33] J. A. Nieminen, A. P. Sutton, J. B. Pethica, Static junction growth during frictional sliding of metals, Acta Metallurgica et Materialia 40 (1992) 25032509.

[34] A. P. Sutton, R. W. Balluffi, Interfaces in Crystalline Materials, Monographs on the physics and chemistry of materials, Clarendon Press, 1995.

[35] S. Kibey, J. B. Liu, D. D. Johnson, H. Sehitoglu, Generalized planar fault energies and twinning in $\mathrm{Cu}-\mathrm{Al}$ alloys, Applied Physics Letters 89 (2006) 191911.

[36] M. Benoit, N. Tarrat, J. Morillo, Density functional theory investigations of titanium $\gamma$-surfaces and stacking faults, Modelling and Simulation in Materials Science and Engineering 21 (2013) 015009.

[37] J. A. Yasi, T. Nogaret, D. R. Trinkle, Y. Qi, L. G. H. Jr, W. A. Curtin, Basal and prism dislocation cores in magnesium: comparison of first-principles and embedded-atom-potential methods predictions, Modelling and Simulation in Materials Science and Engineering 17 (2009) 055012. 
[38] J. A. Yasi, L. G. Hector Jr., D. R. Trinkle, Prediction of thermal cross-slip stress in magnesium alloys from direct first-principles data, Acta Materialia 59 (2011) 5652-5660.

[39] X. Wu, R. Wang, S. Wang, Generalized-stacking-fault energy and surface properties for hcp metals: A first-principles study, Applied Surface Science 256 (2010) 3409-3412.

[40] P. Kwasniak, M. Muzyk, H. Garbacz, K. J. Kurzydlowski, Influence of c, $\mathrm{h}, \mathrm{n}$, and o interstitial atoms on deformation mechanism in titanium-first principles calculations of generalized stacking fault energy, Materials Letters 94 (2013) 92-94.

[41] R. G. Hennig, T. J. Lenosky, D. R. Trinkle, S. P. Rudin, J. W. Wilkins, Classical potential describes martensitic phase transformations between the $\alpha, \beta$, and $\omega$ titanium phases, Physical Review B 78 (2008) 054121-10.

[42] C. Domain, Ab initio modelling of defect properties with substitutional and interstitials elements in steels and zr alloys, Journal of Nuclear Materials 351 (2006) 1-19.

[43] Y. Udagawa, M. Yamaguchi, H. Abe, N. Sekimura, T. Fuketa, Ab initio study on plane defects in zirconium-hydrogen solid solution and zirconium hydride, Acta Materialia 58 (2010) 3927-3938.

[44] T. Nogaret, W. A. Curtin, J. A. Yasi, L. G. Hector Jr, D. R. Trinkle, Atomistic study of edge and screw $\langle c+a\rangle$ dislocations in magnesium, Acta Materialia 58 (2010) 4332-4343.

[45] A. Serra, R. C. Pond, D. J. Bacon, Computer simulation of the structure and mobility of twinning disclocations in h.c.p. metals, Acta Metallurgica et Materialia 39 (1991) 1469-1480.

[46] A. N. Stroh, Dislocations and cracks in anisotropic elasticity, Philosophical Magazine 3 (1958) 625-646.

[47] Z. Wu, W. A. Curtin, The origins of high hardening and low ductility in magnesium, Nature 526 (2015) 62-67.

[48] J. F. Stohr, J. P. Poirier, Etude en microscopie electronique du glissement pyramidal $\{11 \overline{2} 2\}\langle 11 \overline{2} 3\rangle$ dans le magnesium, Philosophical Magazine 25 (1972) 1313-1329. 
[49] T. Obara, H. Yoshinga, S. Morozumi, $\{11 \overline{2} 2\}\langle\overline{1} \overline{1} 23\rangle$ slip system in magnesium, Acta Metallurgica 21 (1973) 845-853.

[50] S. R. Agnew, J. A. Horton, M. H. Yoo, Transmission electron microscopy investigation of $\langle c+a\rangle$ dislocations in Mg and $\alpha$-solid solution Mg-Li alloys, Metallurgical and Materials Transactions A 33 (2002) 851-858.

[51] M. H. Yoo, C. T. Wei, Slip modes of hexagonal-close-packed metals, Journal of Applied Physics 38 (1967) 4317-4322.

[52] Z. Wu, W. A. Curtin, Mechanism and energetics of $\langle c+a\rangle$ dislocation crossslip in hcp metals, Proceedings of the National Academy of Sciences 113 (2016) 11137-11142.

[53] Z. Wu, W. A. Curtin, Brittle and ductile crack-tip behavior in magnesium, Acta Materialia 88 (2015) 1-12. 
